\title{
A woman with a 13-year history of anaplastic thyroid cancer
}

\author{
Piotr Stanisław Szczęsny', Grażyna Orlicz-Szczęsna² \\ ${ }^{1}$ Medical Faculty, Medical University of Gdansk, Gdansk, Poland \\ ${ }^{2}$ Medical Centre - Endocrinology Polyclinic, Lublin, Poland
}

Key words: anaplastic thyroid carcinoma; treatment; survival

A 74-year-old woman came to the Endocrinology Polyclinic in April 2008 due to pain in the front part of the neck, an increase in body temperature to $38^{\circ} \mathrm{C}$, muscle and joint pain, hoarseness, and general weakness that had been present for several days. She had not been treated or diagnosed for thyroid disease. At that time, the patient noticed a sudden, significant enlargement of the right lobe of the thyroid with a large tumour. She also felt shortness of breath, which intensified with physical exertion. In a medical interview, she reported that she had been suffering from type 2 diabetes, arterial hypertension, glaucoma, and degenerative changes in the spine. The thyroid ultrasound revealed a significantly enlarged right lobe of the thyroid with a polycyclic tumour $6 \mathrm{~cm}$ size, containing a large calcification of about $2 \mathrm{~cm}$ on its periphery. There were no focal changes in the left lobe (of the correct size).

Fine-needle aspiration biopsy revealed a necrotic mass with numerous atypical cells.

Laboratory tests revealed euthyroidism, microcytic anaemia $(\mathrm{Hb}-10.9 \mathrm{~g} \%)$, increased erythrocyte sedimentation rate $-59 \mathrm{~mm} / \mathrm{h}$ and leucocytosis - 12,600. The patient was referred urgently for thyroid surgery. Complete excision of the right lobe and subtotal resection of the left lobe was performed. Due to the tumour infiltration of the surrounding tissues, including the trachea, the strumectomy procedure was oncologically non-radical. G3 undifferentiated (anaplastic) thyroid carcinoma (ATC) was diagnosed with histopathology, with metastases to the lymph nodes in the state of partial necrosis. The immunohistochemical reaction for cytokeratin (CK) and epithelial membrane antigen (EMA) was positive. Extensive cellular polymorphism, marked nuclear atypia, numerous abnormal division patterns, and small lymphocyte clusters between neoplastic cells have been described in postoperative preparations. The features of squamous epithelium differentiation, anaplastic cell field with visible division figures, and with lymphocyte inflammatory infiltration were visible (Fig. 1, 2).

After the surgery, the patient's dyspnoea disappeared. Thyroxine substitution (137-150 ug) was initiated, with good hormone dose tolerance. The patient was referred to the Institute of Oncology in Gliwice, where further diagnostics of the advancement of the neoplastic disease was performed and specialistic treatment was started. Undifferentiated thyroid cancer was defined as T4N1bMx in the patient.

A month later, neck and chest computed tomography revealed metastatic right paratracheal lymph nodes near the tumour bed and level II B lymph nodes suspicious for metastasis in the right neck. A mass (diameter $14 \mathrm{~mm}$ ) was also noted in the tumour bed of the left lobe. On targeted biopsy the lymph nodes were positive for malignancy, while the specimen from the left lobe mass revealed the presence of colloid and dispersed normal thyroid cells.

Postoperative scintigraphy showed a higher tracer uptake in this region with no other areas of radiotracer uptake.

The case of this patient was presented to the Thyroid Tumour Group at the Institute of Oncology in Gliwice. Due to the non-radical surgical procedure (because of the infiltration of adjacent tissues, including the trachea) and the histopathological nature of the tumour, it was decided to attempt treatment with an ablative-therapeutic dose of radioactive iodine $\left({ }^{131} \mathrm{~J}\right)$ in terms of possible assessment of loco-regional iodine uptake.

The patient received iodine- $131\left({ }^{98 \mathrm{~m}} \mathrm{Ci}\right) 3$ times every 6 months, after stimulation with thyrotropin alfa. In November 2008, she was referred for neck radiotherapy (5600 cGy/g over 6 weeks). Follow-up scintigraphy in October 2009 revealed no radiotracer uptake. 


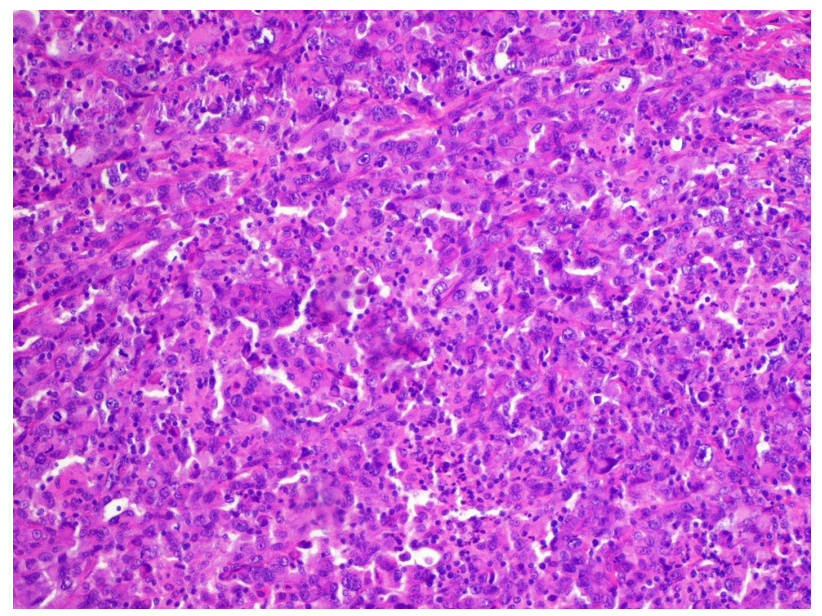

Figure 1. Anaplastic thyroid cancer. Fields of anaplastic cells, division figures visible. Haematoxylin and eosin staining $(\times 100)$

Currently, the patient is 87 years old. A follow-up ultrasound of the neck area shows a volume area of about $0.6 \mathrm{~mL}$ with the features of a fibrotic stump in the left lobe bed. She does not report local or general complaints related to thyroid neoplastic disease, and she undergoes routine medical check-ups.

Anaplastic thyroid carcinoma is a very malignant type of neoplasm with poor prognosis, with mortality reaching $100 \%$ within a few months.

It arises as a primary tumour from undifferentiated thyroid cells or following progression of well-differentiated papillary or follicular carcinoma [1].

Molecular aberrations are implicated in the pathogenesis, with the TP53 gene mutation as the main underlying mechanism [2]. Patients are almost always euthyroid. The mean age at diagnosis is 65 years. The disease course is characterized by aggressive tumour growth and local invasion of the surrounding structures [3]. Histologically, the tumour is composed of highly anaplastic giant, polymorphic, or spindle-shaped cells. Necrotic changes in the tumour occur rapidly, with macrophage infiltrates. Distant metastasis of cancer is frequent, but death usually occurs as a result of damage to vital structures within the neck [4]. Most often, ATC does not produce thyroglobulin, nor does it accumulate radioactive iodine [5]. In the treatment of this type of cancer (ATC), thyroid resection, possibly complete, and external teleradiotherapy are recommended. In recent years, trials of pharmacotherapy have been reported. However, chemotherapy does not show the expected positive therapeutic effects [6, 7]. Ferrari et al. [8] emphasize the important role of molecular tests in selecting the form of therapy in each patient with ATC. Despite many studies on this cancer, the current treatment strategy for anaplastic thyroid cancer is still insufficient for significant extension of the

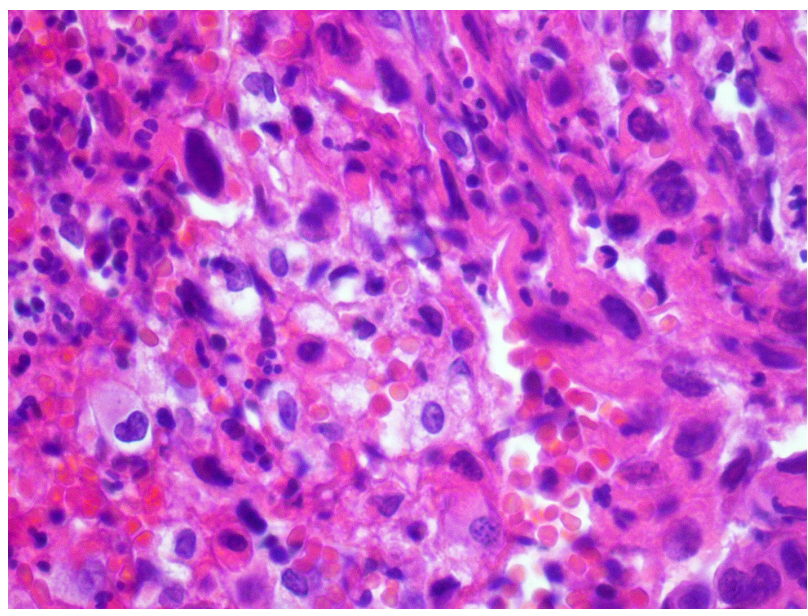

Figure 2. Anaplastic thyroid cancer. Anaplastic cell fields with lymphocyte inflammatory infiltration, features of squamous cell differentiation. Haematoxylin and eosin staining $(\times 200)$

patient's life [9]. Based on the case of an elderly woman with 13 years of ATC (still alive, without evidence of an active neoplastic disease), a thesis can be made that a very individually selected form of therapy for this cancer is necessary. In this case, the strumectomy (non-radical), as well as external radiotherapy and 131-J therapy gave a good effect in the form of very long survival of this patient with histopathologically confirmed ATC.

\section{Conflict of interest}

None declared.

\section{References}

1. Maitra A. Układ dokrewny. In: Kumar V, Abbas AK, Aster JC. ed. Robbins Patologia. Edra Urban\&Partner, Wrocław 2019: 892-895.

2. Drewa G, Ferenc T. Medical genetics [in Polish]. Elsevier Urban\&Partner, Wrocław 2011: 148-152.

3. Daroszewski J, Paczkowska K, Jawiarczyk-Przybyłowska A, et al. Anaplastic thyroid carcinoma with rapie thyrotoxicosis - a case report and the literature review. Endokrynol Pol. 2018; 69(1): 28-31, doi: 10.5603/EP.a2018.0010, indexed in Pubmed: 29319130.

4. Abe I, Lam AKY. Anaplastic thyroid carcinoma: Updates on WHO classification, clinicopathological features and staging. Histol Histopathol. 2021; 36(3): 239-248, doi: 10.14670/HH-18-277, indexed in Pubmed: 33170501.

5. Oh JiM, Baek SeH, Gangadaran P, et al. A Novel Tyrosine Kinase Inhibitor Can Augment Radioactive Iodine Uptake Through Endogenous Sodium/Iodide Symporter Expression in Anaplastic Thyroid Cancer. Thyroid. 2020; 30(4): 501-518, doi: 10.1089/thy.2018.0626, indexed in Pubmed: 31928162

6. Jarząb B, Dedecjus M, Słowińska-Klencka D, et al. Guidelines of Polish National Societies Diagnostics and Treatment of Thyroid Carcinoma. 2018 Update. Endokrynol Pol. 2018; 69(1): 34-74, doi: 10.5603/EP.2018.0014, indexed in Pubmed: 29442352

7. Mirabile A, Biafora M, Giordano L, et al. Uncommon Site of Metastasis and Prolonged Survival in Patients with Anaplastic Thyroid Carcinoma: A Systematic Review of the Literature. Cancers (Basel). 2020; 12(9), doi: 10.3390/cancers12092585, indexed in Pubmed: 32927794.

8. Ferrari SM, Elia G, Ragusa F, et al. Novel treatments for anaplastic thyroid carcinoma. Gland Surg. 2020; 9(Suppl 1): S28-S42, doi: 10.21037/gs.2019.10.18, indexed in Pubmed: 32055496.

9. Lennon P, Deady S, Healy ML, et al. Anaplastic thyroid carcinoma: Failure of conventional therapy but hope of targeted therapy. Head Neck. 2016; 38 Suppl 1: E1122-E1129, doi: 10.1002/hed.24170, indexed in Pubmed: 26879282 